\title{
Morphological tools for range-interval segmentation of elastic lidar signals
}

\author{
Francesc Rocadenbosch, Michaël Sicard, Mohd Nadzri Md Reba, and Sergio Tomás \\ Universitat Politècnica de Catalunya (UPC), Dep. of Signal Theory and Communications (TSC), \\ Remote Sensing Lab (RSLAB), C/Jordi Girona, 1-3, D4-016, \\ 08034 Barcelona, SPAIN. \\ roca@tsc.upc.edu
}

\begin{abstract}
This article presents a preliminary semi-automated range-interval segmentation toolset for the identification of: 1) the apparent range of full overlap, 2) the clear-sky level (i.e., the molecular level), and cloud layers (cloud-base, cloud-peak, and cloud-top range), and 3) apparent homogeneous extinction intervals.
\end{abstract}

Keywords- lidar, ceilometer, clouds, segmentation, pattern recognition.

\section{INTRODUCTION}

Inversion of atmospheric optical parameters (namely, extinction and backscatter) from elastic/Raman lidar records involves different preprocessing steps such as segmentation of the return signal into different range intervals [1]

Identification of spatial ranges in range-corrected signal records with different morphological characteristics such as the starting range of full overlap factor (OVF) -between the laser beam and the telescope FOV (field of view)-, the end of the boundary layer, cloud or aerosol layers aloft is by itself a final user product, even when these ranges are just approximated ones. An important added value arises when this range-interval segmentation can ideally be automated or operated with reduced human intervention on time-series of power returns, as is the case of cloud monitoring and diurnal/nocturnal-cycle time plots.

Spatial segmentation of elastic signals is also of application in the so-called slice method [2], in which the atmosphere is divided into layers where the well-known slope method or, alternatively, exponential-curve fitting methods [3] are successively applied to adjacent range-intervals of homogeneous characteristics in order to retrieve a piece-wise range-dependent extinction estimate. Likewise, inherent inhomogeneous inversion algorithms such as the Klett's method and its variants [4] can be applied to different range sub-intervals of the whole inversion range with different calibrations and correlating hypotheses in each one of these segments.

This paper is organised as follows: Sect. II presents key signal processing methods, Sect. III the identification of the apparent range of full overlap, the molecular clear-sky level, and cloud layers, and Sect. IV, that of apparent aerosol homogeneous extinction intervals. Sect. V gives conclusion remarks.

\section{PROCESSING FunCTIONS}

Identification of the above mentioned morphological characteristics from lidar returns (Fig. 1) requires combination of signal-processing methods both analytical (filters) and nonanalytical such as search-and-sort procedures, as well as the inclusion of "a priori" information from the user's side (e.g. existence of low clouds, minimum spatial resolution desired, etc.). Besides, some degree of parameter tuning of the different default setting parameters is required for the typology of lidar scenes can be very different from one set of records to another.

Thus, low/high pass filtering of the range-corrected (noisecorrupted) lidar return is respectively used to obtain a virtually noise-cancelled smoothed power estimate (Fig. 2a,b) and a "shape" distortion indicator (Fig. 2c). The low-pass smoothed lidar return (Fig. 2a,b) is formed as

$$
Y(\mathrm{R})=F_{L P}\left[\mathrm{R}^{2} Z(\mathrm{R})\right],
$$

where $Z(R)$ is the noise-corrupted power return (i.e., $Z(R)=P(R)+n(R)$ with $P(R)$ the noiseless power component and $n(R)$ the observation noise), $\mathrm{R}$ is the range, and $F_{L P}$ is a lowpass filter operator. For example, a $3^{\text {rd }}$-order Butterworth zerolag digital filter with a Nyquist-normalised cut-off frequency, $f_{c}$, in the $0.05-0.1$ range, usually provides low distortion, acceptable spatial resolution, and high noise rejection for most scenes with a similar signal-to-noise ratio (SNR) to that of Fig.1. An initial selection criterion for $f_{c}$ is to estimate the power espectral density (PSD) of the lidar return so as to ensure that e.g. $85 \%$ of the power is retained though, as mentioned above, this is just a reasonable tuning criterion, which finally relies on the user's side.

The "shape" distortion indicator due to low-pass filtering (Fig. 2c) is formed as

$$
\varepsilon_{f}(R)=R^{2} Z(R)-Y(R)
$$

Note that this is equivalent to high-pass filtering the rangecorrected lidar return, $R^{2} Z(R)$, and that in the ideal case that all the low-frequency contents of the lidar signal concentrated within the low-pass filter's bandwidth, $Y(R)$ would basically retain the noiseless signal component, $Y(\mathrm{R}) \approx R^{2} P(R)$, and $\varepsilon_{f}(R)$ be zero-mean range-corrected white noise, $\varepsilon_{f}(R)=R^{2} n(R)$. The 
filtering procedure may be repeated over a particular analysis interval (this time with a higher cut-off frequency, $f_{c}$ ), if $\varepsilon_{f}$ indicator substantially departs from white noise.

A main search-and-sort procedure is applied to low-pass signal $Y(R)$ along each successive sample to classify candidate relative maxima and minima. Particularly in the far-range, where the SNR is poorer and noise may well cause false singular points, they will be statistically validated via threshold limiting $\varepsilon_{f}(R)$ (Fig. $2 b$ in Sect. III.C).

\section{OVF, CLEAR-SKY LEVEL AND CLOUD DETECTION ALGORITHMS}

\section{A. $O V F$}

The overlap function can be estimated from the field-ofview of the receiving telescope, the divergence of the laser and the geometry of the lidar system [5], and can be experimentally determined by Raman techniques [6].

What is determined here, from just the lidar return and the morphological tools being presented is the apparent starting range of full overlap, $R_{\text {ovf }}$ (Fig. 2a). It is estimated as the first absolute maxima of $Y(R)$, in a user-defined search interval, $I=\left[0, R_{0}\right] \quad$ (e.g., $\quad R_{0}=0.3$ and $2 \mathrm{~km}$ for a low/far-range tropospheric lidar system, respectively). Formally,

$$
R_{\text {ovf }}=\max \left\{R_{\max , i} \in I\right\}, \quad I=\left[0, R_{0}\right] .
$$

Simulations of the overlap function applied to lidar returns show that the estimated first absolute maximum in bi-static lidar occurs at a range shorter than the true full-overlap range [7].

\section{B. Clear-sky level identification}

The clear-sky level is defined as the signal from molecular scattering only. From the US-standard atmosphere model (at the present state of research only standard conditions have been tested) this follows a well-known exponential-like decreasing trend.

To determine it from the set of available $Y(R)$ relative minima (Sect. II), the clear-sky minima selected from this set must follow the monotonically decreasing order relation

$$
R_{i}<R_{i+1} \Rightarrow Y\left(R_{i}\right)>Y\left(R_{i+1}\right)+\delta_{i} ; \quad \delta_{i}=\max \left[\varepsilon_{f}(R)\right]_{I_{i}}, I_{i}=\left[R_{i}, R_{i+1}\right] \text {, }
$$

where $R_{i}$ and $R_{i+1}$ are respectively the ranges corresponding to two successive clear-sky minima, and $\delta_{i}$ is a noise-threshold correction term. In range intervals where SNR $>3$ approximately, $\delta_{i}$ can be disregarded.

According to (4), Fig. 2a depicts clear-sky minima in grey circles and rejected ones (from the initial search-and-sort procedure of Sect. II) in crosses. Thus, minima located on the falling slope of the cloud (range interval [4.8, 5.2] km, Fig. 2a) fail (4) test when they are compared with point $\mathrm{D}$, which is their previous clear-sky absolute minimum.

\section{Cloud-detection algorithm}

Here, we mainly follow Pal, Steinbrecht and Carswell's definitions [8]:

1) Cloud base: "The cloud base, $\mathrm{Rb}$, is defined as a local minima belonging to the clear-sky level at the beginning of the sudden increase of the backscattered signal over the ambient air return." (D in Fig. 2b).

2) Cloud top: "The apparent cloud-top height, Rt, -we say "apparent" here because for large optical depths the emitted laser pulse may completely be attenuated before the cloud top is reached- requires that at $\mathrm{Rt}$ the backscattered signal is less than or equal to the backscattered signal at the cloud base Rb". This translates into the condition (E in Fig.2b)

$$
Y\left(R_{t}\right)<Y\left(R_{b}\right) .
$$

3) Separate cloud layers: "Condition for two peaks to indicate separate cloud layers is that a layer of clear air must exist between them."

4) Cloud peak: We define the cloud peak as the absolute maximum (from the search-and-sort procedure of Sect. II) in the cloud inteval $\left[R_{b}, R_{t}\right]$.

By relating definitions 1-2 above to (4), we note that the cloud base, $R_{b}$, and the cloud top, $R_{t}$, are always two successive clear-sky level points (i.e., $R_{i}=R_{b}, R_{i+1}=R_{t}$ ) and that (5) is identical to (4) except for the noise-threshold correction term, $\delta_{i}$.

In the presence of clouds, high-frequency components associated to fast lidar signal transients cause that $\delta_{i}$ is dominated by the smoothing error between the range-corrected lidar observable and its smoothed version $Y(R)$ (Fig. 2b). As a result, the approximation $\varepsilon_{f}(R) \approx R^{2} n(R)$ (Sect. II) collapses and $\delta_{i}$ largely increases. $\delta_{i}$ is thus of advantage in low-SNR range intervals ([F $G]$ in Fig. 1), where noise makes difficult to precisely assess the cloud-top height ("G") unless additional threshold-decision criteria are introduced. One of them consists of comparing $\delta_{i}$ (equivalently, the noise standard deviation of $\left.\varepsilon_{f}(R), \sigma\right)$ with both the mean and maximum values of $Y(R)$. This is, however, a matter of research, particularly, in terms of probability of detection and false alarm.

Definitions 1-4 above are at the ground of most ceilometer algorithms [8][9][10] and a signal processing examples is shown on Fig. 3.

\section{IDENTIFICATION OF HOMOGENEOUS-SLOPE INTERVALS (HSI)}

It is well known from solution of Bernouilli's differential lidar equation that there are returns from inhomogeneous atmospheric conditions that cannot be distinguished from those of homogeneous conditions unless "a priori" information (cooperative sensors) is provided [11]. Therefore, we will derive now an algorithm to identify intervals with apparent constant 
extinction (i.e., with negative constant slope over $\left.Y(\mathrm{R}) \approx R^{2} P(R)\right)$.

HSI are delimited not only by clear-sky points (Sect.III.B) but also by corner points. These are "angular" points ("N" in Fig. 4) for which the function curvature, $K$, reaches a maximum. Following [12], they can be computed as

$$
\max \{K\} \approx \max \left\{\frac{d^{2} Y}{d R^{2}}\right\} .
$$
(4).

As a result, corner points are included in the sorted set of

From this set of candidate points, the algorithm searches for HSIs compliant with: 1) a user-defined minimum differential change in slope from one interval to another, $\varepsilon_{s l p}$, so that

$$
\left|m_{1}-m_{2}\right| \geq \varepsilon_{s l p}
$$

where $m_{1}$ and $m_{2}$ are respectively the interval slopes, and 2) a user-defined minimum interval size (search spatial resolution),

$$
\Delta=\left|R_{i}-R_{i-1}\right| \text {. }
$$

Fig. 4 shows four successive steps of the algorithm operation. At each succeeding step, three singular points are considered (for example, $\mathrm{M}, \mathrm{N}$ and $\mathrm{O}$ in Fig. 4a), which determine range subintervals ( $\mathrm{MN}$ and $\mathrm{NO}$ ), where slopes $m_{1}$ and $m_{2}$ are computed. The connection point ("N" in Fig. 4a, " $\mathrm{O}$ " in Fig. 4b, etc.) is tested according to (7) above. If the test is passed then the target point becomes a border point between two different HSIs, otherwise the point is deleted from the set and, consequently, the two intervals merge into a single larger one (Fig. 4c). In this event, the current interval slope (NP in Fig. 4c) is compared with that of the next one (NR in Fig. 4c) but now using $\varepsilon_{s l p}^{\prime}=\varepsilon_{s l p} / 2 . \varepsilon_{s l p}$ is not reset to its initial default value until the current HSI is validated.

\section{CONCLUSIONS AND FUTURE WORK}

Three main algorithms have been presented for the determination of the apparent OVF, clear-sky level and cloud layers, and HSIs based from the morphology of elastic lidar returns. Signal processing methods rely on both low/high pass filtering of the range-corrected signal, advanced search-andsort computational procedures, and a minimum set of userdefined "a priori" information concerning decision threshold defaults.

Future work is to concentrate on adaptive segmentation decision rules combining both the current and past processed records, inclusion of "a priori" settings from scene classification, and most important, verification with cooperative sensors.

\section{ACKNOWLEDGMENT}

The authors wish to acknowledge the following entities for partially supporting this research work and lidar systems developed at UPC: European Union and FEDER funds under the EARLINET-ASOS (EU Coordination Action) contract $n^{\circ}$ 025991 (RICA), and (EU Specific Support Action) contract $n^{\circ}$ 011863 (RIDS): "Technology development programme towards a European Extremely Large Telescope"; MCYT (Spanish Ministry of Science and Technology) and FEDER funds under the projects TEC2006-07850/TCM and REN200309753-C02-02, Complementary Actions CGL2006-26149E/CLI, CTM2006-27154-E/TECNO, and Special Action REN2002-12784-E; MITYC (Spanish Ministry of Industry, Tourism and Commerce) under the PROFIT project, CIT020400-2005-56. MCYT is also thanked for the Ramón y Cajal position hold by Dr. M. Sicard, and Local Government of Catalonia (Generalitat de Catalunya/AGAUR) for Mr. Md. Reba's predoctoral fellowship.

\section{REFERENCES}

[1] G. Pappalardo, A. Amodeo, M. Pandolfi, U. Wandinger, A. Ansmann, J. Bösenberg, V. Matthias, V. Amiridis, F. De Tomasi, M. Frioud, M. Iarlori, L. Komguem, A. Papayannis, F. Rocadenbosch, X. Wang. "Aerosol lidar intercomparison in the framework of the EARLINET project. 3. Raman lidar algorithm for aerosol extinction, backscatter and lidar ratio." Appl. Opt., 43 (28), 5370-5385 (2004).

[2] R.T. Brown, "A New Lidar for Meteorological Application,” J. Appl. Meteorol. 12, 698-708 (1973)

[3] F. Rocadenbosch, A. Comerón and D. Pineda, "Assessment of lidar inversion errors for homogeneous atmospheres," Appl. Opt., 37(12), 2199-2206, (1998).

[4] J.D. Klett, "Lidar Inversion with Variable Backscatter/Extinction Ratios," Appl. Opt. 24, 1638-1643, (1985).

[5] R.M. Measures, Laser Remote Sensing: Fundamentals and Applications, (Krieger, Malabar, Fla., 1992), Chap.7, pp. 256-265.

[6] U. Wandinger and A. Ansmann, "Experimental Determination of the Lidar Overlap Profile with Raman Lidar," Appl. Opt. 41, 511-514 (2002).

[7] R. Velotta, B. Bartoli, R. Capobianco, L. Fiorani, and N. Spinelli, "Analysis of the Receiver Response in Lidar Measurements," Appl. Opt. 37, 6999-7007 (1998).

[8] S.R. Pal, W. Steinbrecht, A. I. Carswell, "Automated method for lidar determination of cloud-base height and vertical extent," Appl. Opt. 31 (10), (1992).

[9] C. Münkel, "Cloud base height determination in rain, snow, and fog with a low-cost eye-safe lidar," SPIE Vol. 2506, 535-542 (1995).

[10] J. Streicher, C. Werner, F. Köpp, "Verification of lidar visibility, cloud base height, and vertical velocity measurements by laser remote sensing," SPIE Vol. 2506, 576-579 (1995).

[11] G.L. Kunz, "Probing of the Atmosphere with Lidar," Proc. Remote Sensing of the Propagation Environment (AGARD-CP-502). Conf. Date: 30 Sept.-4 Oct. 1991, Conf. Location: Cesme, Turkey. Publisher: AGARD, Neuilly sur Seine, France, 23, 1-11 (1992).

[12] I. Bronshtein, K. Semendiaev, Part 3, Sec. 2A, Differential Geometry in "Manual of Mathematics for Engineers and Students" (in Spanish). Ed. Mir, (1993). 


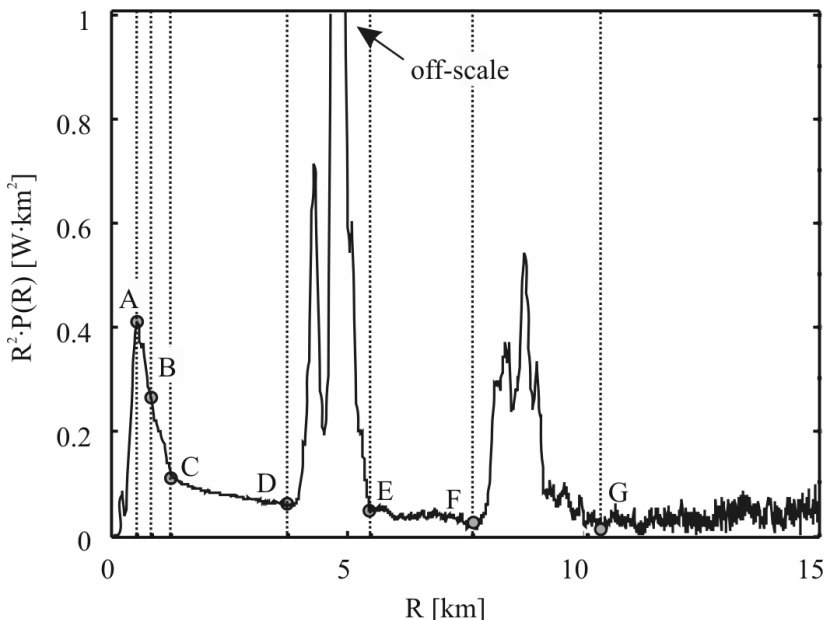

Figure 1. Partitioned range-corrected backscatter lidar signal.

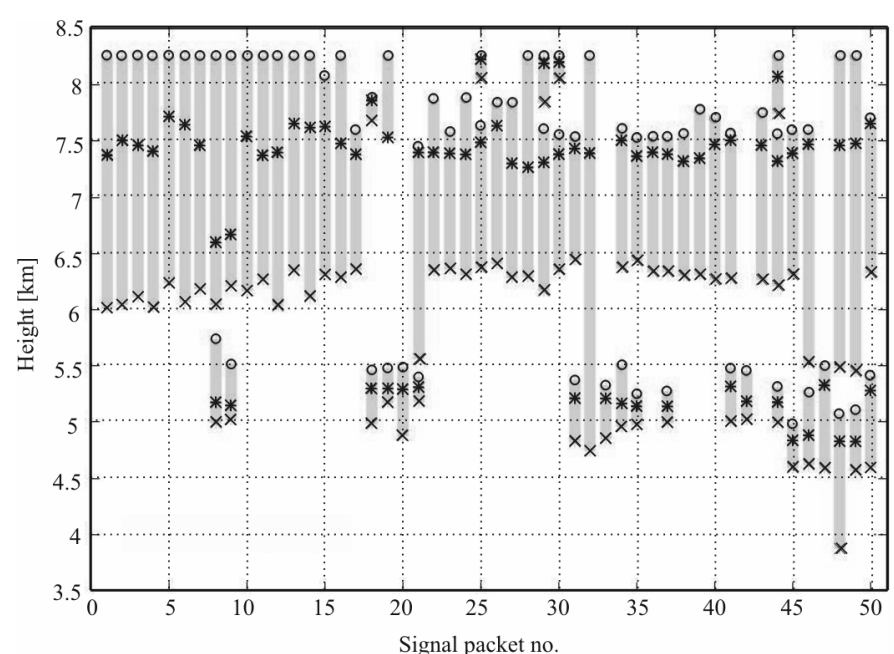

Figure 3. Identification of the apparent cloud-base, -peak, and -top heights.
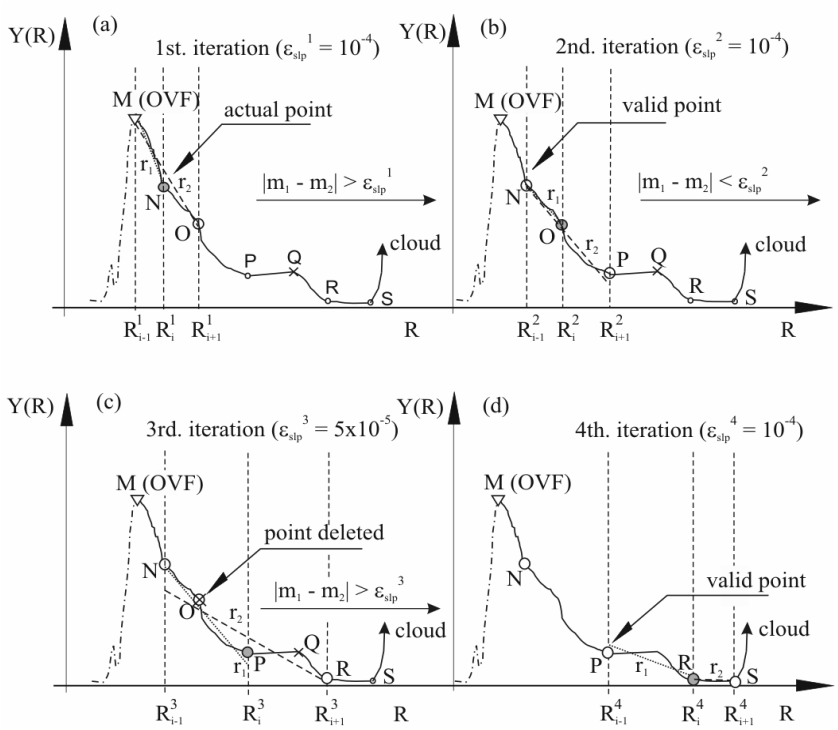

Figure 4. Identification of homogeneous slope intervals (HSI).

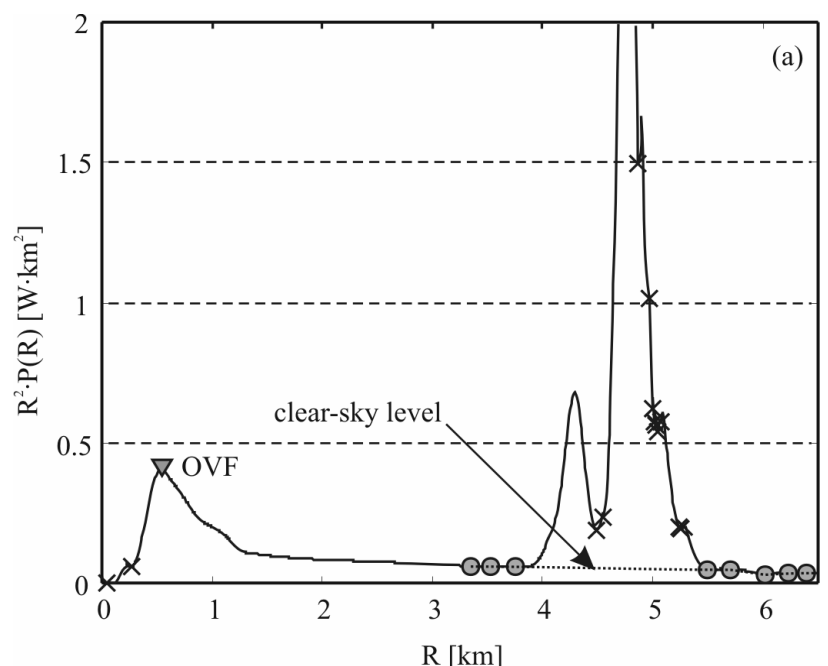

Figure 2. Processing functions, clear-sky level, and cloud layer identification. (a) (solid trace) Low-pass smoothed return (1), (dotted) clear-sky level.

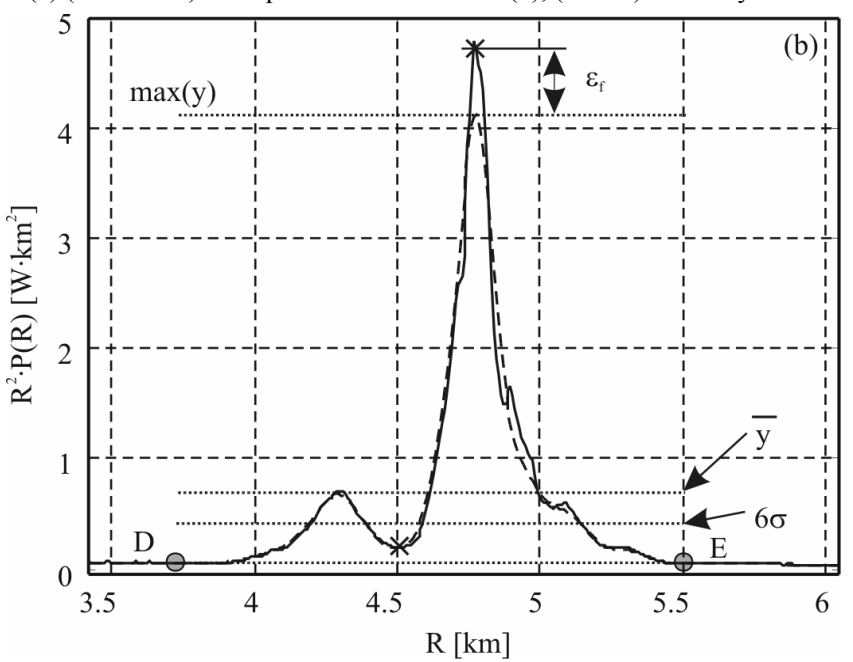

(b) Identification of a cloud layer in the $[3.42,6.04] \mathrm{km}$ interval of Fig. 1. (Solid trace) Observable lidar return, (dotted) smoothed version (1).

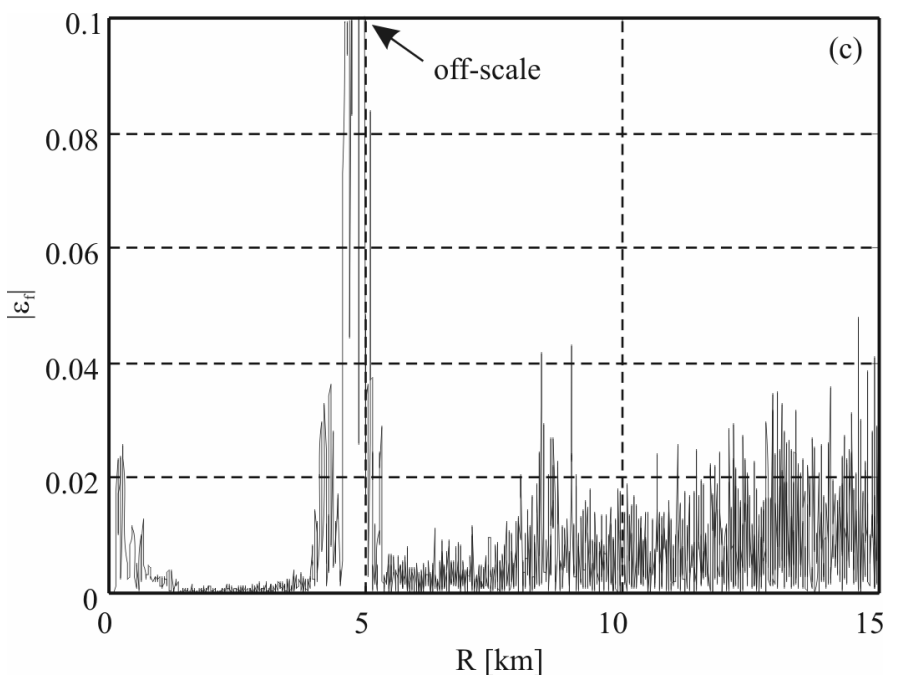

(c) Error function, $\varepsilon_{\mathrm{f}}(\mathrm{R})$, for Fig. 1, (2). 